\title{
Improved Slice Method for Slope Stability Analysis under Load
}

\author{
Yanjie Sun *
}

* 747720426@qq.com; Tel.:+8615891763959(Xi'an University of Science and Technology, Shaanxi, Xi'an, 710054)

\begin{abstract}
The load on the top of a slope is an important cause of slope failure, and it is of great significance to study the relationship between the load and the stability of the slope. This paper uses elastic theory and Moore Coulomb's theory as transformation conditions to obtain the slope stability coefficient expression under slope top load based on the Swedish slice method. In view of the actual engineering, the corresponding slope model structure was established, and 5 sliding surfaces were set with the crack on the top of the slope as the shear outlet. According to the slope stability coefficient expression, the stability coefficient of the set sliding surface is solved. The result shows that the slope is unstable under the load. The judgment result is consistent with the GEO-STUDIO check calculation result. This method can provide reference for theory and engineering practice.
\end{abstract}

Keywords: loess slope; shallow landslide;Slice method; Moore Coulomb theory; slope stability coefficient

\section{Introduction}

The external load on the top of the slope is the main cause of slope instability [1-4].Under the action of the upper load,the slope will produce a slip surface that does not pass through or under the slope toe,which increases the risk of slope instability[5].Therefore,studying the slope top load has important theoretical and engineering practical significance for the stability analysis of loess slopes.

In order to solve the above problems, this article combines the Swedish slice method, Moore Coulomb theory and elastic theory to obtain the slope stability evaluation method under the slope top load.

\section{Method introduction}

In the Swedish strip method, the slope is a homogeneous soil,the sliding surface is $\mathrm{AC}$, the center is $\mathrm{O}$, and the radius is $\mathrm{R}$.The sliding body $\mathrm{ABC}$ is divided into several soil strips,as shown in Figure 1.Take any one of the soils (Article i), the self-weight of the soil strip is $\mathrm{Gi}$, then $\mathrm{Gi}=\gamma$ bihi, where is the soil weight, bi and hi are the width and average height of the soil strip,respectively. The Gi-induced sliding surface can be decomposed into the normal stress $\mathrm{Ni}$ passing through the center of the circle and the shear stress $\mathrm{Ti}$ tangent to the sliding arc. Assuming that the angle between the normal line of the ground midpoint of the soil strip and the vertical line is $\theta \mathrm{i}$, then $\mathrm{Ni}=\mathrm{Gicos} \theta \mathrm{i}$ and $\mathrm{Ti}=\mathrm{Gisin} \theta \mathrm{i}$ can be obtained[6]. 


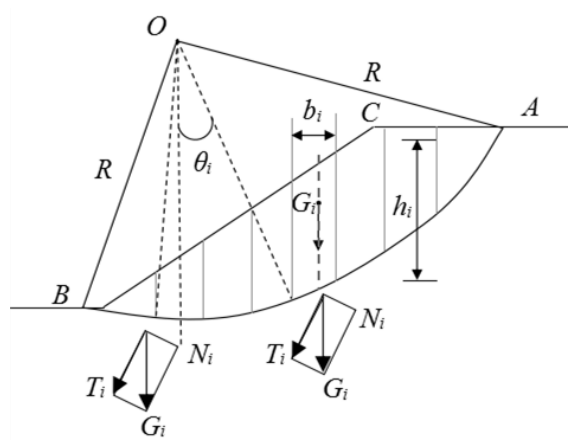

Figure 1. Calculation diagram of slice method.

Suppose the reaction force acting on the normal stress on the bottom of the soil strip is $\mathrm{Ni}^{\prime}$, and its magnitude is equal to and opposite to the normal stress. Suppose the shearing force acting on the bottom of the soil strip is Ti', and its magnitude is the product of the shearing strength on the bottom of the soil and the length of the sliding arc, which is opposite to the sliding direction. When the slope is in a stable state, assuming that the stability coefficient on the sliding surface at the bottom of each soil is equal to the stability coefficient on the entire sliding surface, the shear resistance is

$$
T_{f i}=\frac{\tau_{f i} l_{i}}{K}=\frac{\left(c+\sigma_{i} \tan \varphi\right) l_{i}}{K}=\frac{c l_{i}+N_{i}^{\prime} \tan \varphi}{K}
$$

If the center $\mathrm{O}$ of the sliding door is taken as the moment balance, then $\sum \mathrm{TiR}=\sum \mathrm{TfiR}$, so the slope stability coefficient is

$$
K=\frac{\Sigma\left(c l_{i}+N_{i}^{\prime} \tan \varphi\right)}{\Sigma T_{i}}=\frac{\Sigma\left(c l_{i}+G_{i} \cos \theta_{i} \tan \varphi\right)}{\Sigma G_{i} \sin \theta_{i}}=\frac{\Sigma\left(c l_{i}+\gamma b_{i} h_{i} \cos \theta_{i} \tan \varphi\right)}{\Sigma \gamma b_{i} h_{i} \sin \theta_{i}}
$$

\section{Method for calculating slope stability coefficient}

In order to analyze the slope stability coefficient under the slope top load, this paper assumes that there is a uniformly distributed load $\mathrm{P}$ on the slope top, and the sliding surface is a straight line, as shown in Figure 2.

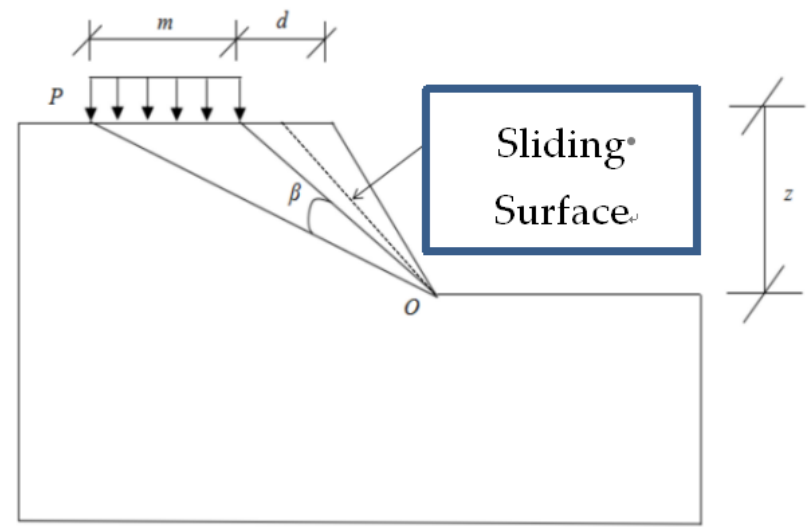

Figure 2. Forces on sliding surface under slope top load.

In the figure, $\mathrm{P}$ is the unit building load per unit length; $\mathrm{M}$ is the size of the building load action surface; $\mathrm{d}$ is the distance between the building load action position and the slope shoulder; o is a certain point on the sliding surface; $\beta$ is the point o to the load action surface The angle between the two endpoints; $\mathrm{z}$ is the vertical distance from the slope surface to a point on the sliding surface. 
When a uniformly distributed strip load acts on the surface of the foundation, the load will change the force at a point below the foundation. In this paper, this point is set as a point on the sliding surface of the slope, and the sliding surface is regarded as a collection of such points. According to the theory of elasticity, the principal stresses at point o are shown in equations (3) and (4)[6].

$$
\begin{aligned}
& \sigma_{1}=\frac{P}{\pi}(\beta+\sin \beta) \\
& \sigma_{3}=\frac{P}{\pi}(\beta-\sin \beta)
\end{aligned}
$$

According to the Moore-Coulomb theory, the shear stress and shear strength at point o are shown in equations (5) and (6) [7].

$$
\begin{gathered}
\tau=\frac{1}{2}\left(\sigma_{1}-\sigma_{3}\right) \sin 2 \alpha^{\prime} \\
\tau_{f}=c+\sigma \tan \varphi \\
\sigma=\frac{1}{2}\left(\sigma_{1}+\sigma_{3}\right)+\frac{1}{2}\left(\sigma_{1}-\sigma_{3}\right) \cos 2 \alpha^{\prime}
\end{gathered}
$$

In the formula, $\mathrm{c}$ is the cohesive force of the soil; $\varphi$ is the internal friction angle of the soil; $a^{\prime}$ is the angle of the principal stress.

Combining the above equations can get the expressions of shear stress and shear strength at point $\mathrm{o}$, as shown in equations (8) and (9).

$$
\begin{gathered}
\tau=\frac{P}{\pi} \sin \beta \sin 2 \alpha^{\prime} \\
\tau_{f}=c+\left(\frac{P}{\pi} \beta+\frac{P}{\pi} \sin \beta \cos 2 \alpha^{\prime}\right) \tan \varphi
\end{gathered}
$$

Supposing o is a square unit and the side length is unit 1, then the principal stress angle is $45^{\circ}$. Equations (8) and (9) can be transformed into equations (10) and (11).

$$
\begin{gathered}
\tau=\frac{P}{\pi} \sin \beta \\
\tau_{f}=c+\left(\frac{P}{\pi} \beta\right) \tan \varphi
\end{gathered}
$$

According to formula (6), the expression of slope stability coefficient under slope top load can be obtained, as shown in formula (12).

$$
K=\frac{\left(G \cos \alpha+\frac{P}{\pi} \int_{\beta_{1}}^{\beta_{2}} x d x\right) \tan \varphi+c L}{G \sin \alpha+\frac{P}{\pi} \int_{\beta_{1}}^{\beta_{2}} \sin x d x}=\frac{\tan \varphi\left[G \cos \alpha+\frac{P}{2 \pi}\left(\beta_{2}+\beta_{1}\right)\left(\beta_{2}-\beta_{1}\right)\right]+c L}{G \sin \alpha+\frac{P}{\pi}\left(\cos \beta_{1}-\cos \beta_{2}\right)}
$$


In the formula, the value of $\beta$ ranges from $\beta 1$ to $\beta 2$. $L$ is the length of the sliding surface.

It can be seen from Figure 2 that $\beta 1$ is located at the top of the slope. Equation (13) can be simplified when $\beta 1$ is $0^{\circ}$.

$$
K=\frac{\tan \varphi\left(G \cos \alpha+\frac{P}{2 \pi} \beta_{2}^{2}\right)+c L}{G \sin \alpha+\frac{P}{\pi}\left(1-\cos \beta_{2}\right)}
$$

\section{Project}

A road under construction is located in the Loess Plateau region of northern Shaanxi. Disasters are frequent in this area. In the K9+470 K9+540 section of the proposed highway, the side slope is ready to be excavated, as shown in Figure 3. However, according to the on-site geological survey, there is a crack at $2 \mathrm{~m}$ from the shoulder of the slope to be excavated. The length of the crack is $5.78 \mathrm{~m}$ and the width is $0.008 \mathrm{~m}$. And there is a high-voltage iron tower $20 \mathrm{~m}$ away from the slope shoulder, and the bottom area of the tower is about $10 \mathrm{~m} 2$. Whether the excavation of the side slope will cause slope instability and threaten the safety of the high-voltage tower is the main consideration for this project, as shown in Figure 4.In order to analyze the stability of the slope, this paper will use the slope stability solving formula under load to calculate and analyze the actual situation of the project. Figure 5 shows the geometry used for calculation.

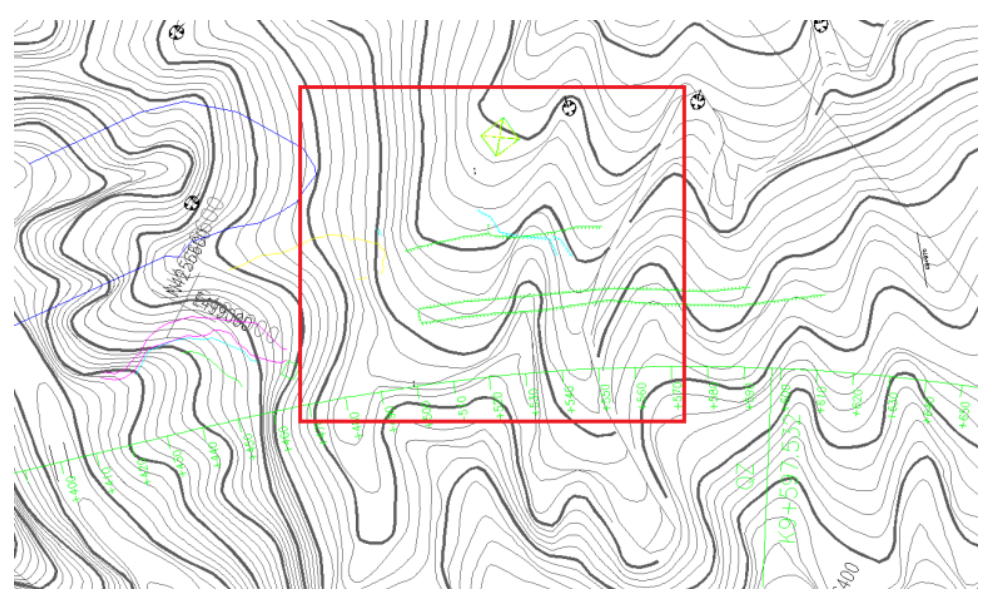

Figure 3. Project geographic location. 


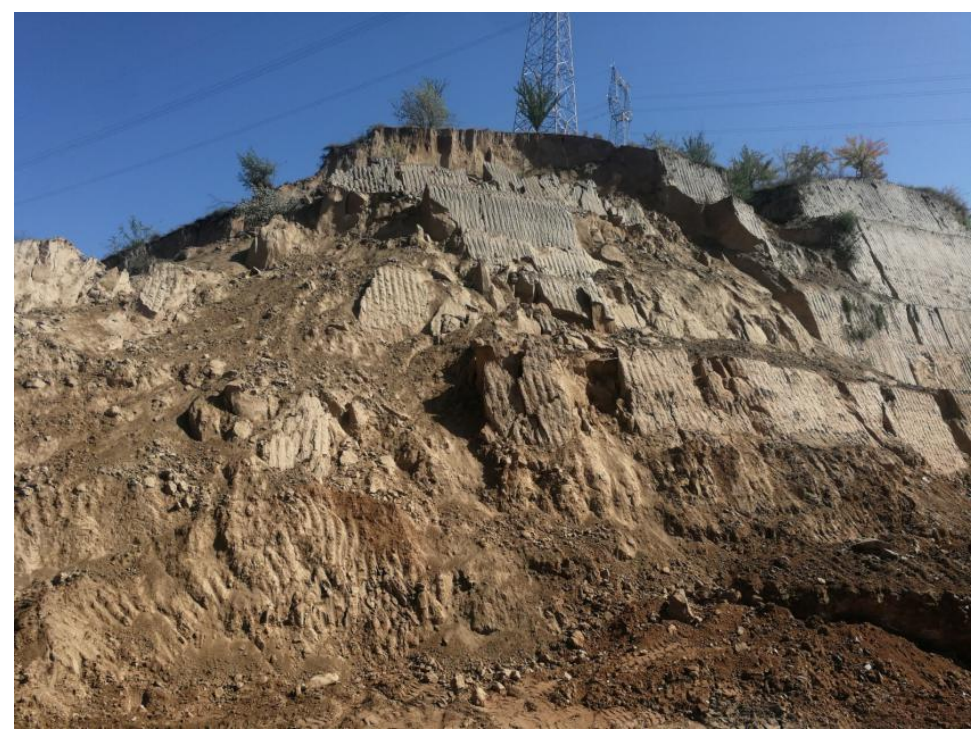

Figure 4. Current status of the slope.

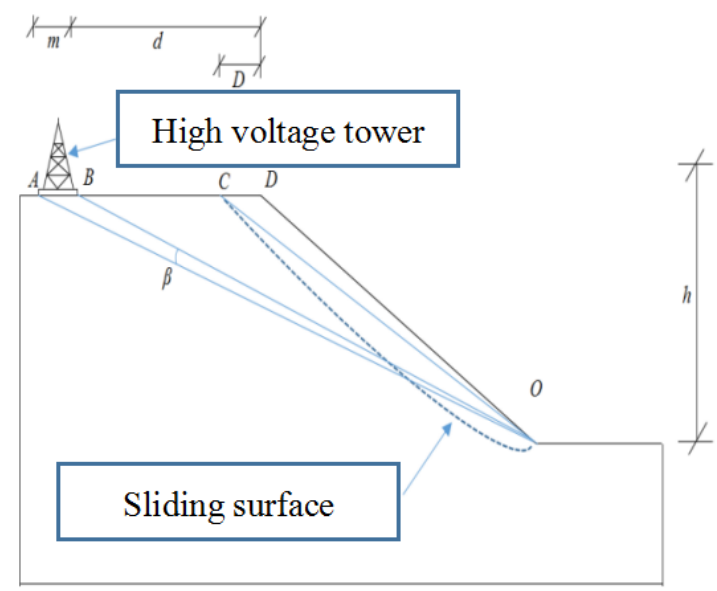

Figure 5. Calculation model.

The loess at the site was drilled, and the core is shown in Figure 6. The physical parameters of the loess are tested, as shown in the following table 1.

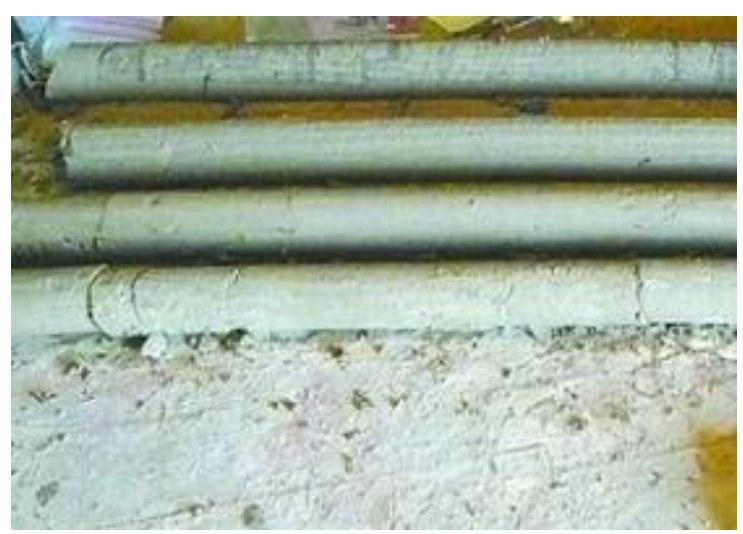

Figure 6. Loess drilled.

Table 1. Values of relevant parameters of slope.

$$
\alpha /\left(^{\circ}\right)
$$

$\mathbf{c}^{\prime} / \mathbf{k P a}$

$\varphi^{\prime} /\left(^{\circ}\right)$

$\mathrm{P} / \mathrm{kPa}$

$\mathbf{M} / \mathbf{m}^{2}$ 
In Figure $4, D=2 m, h=32 m, \beta=7.47^{\circ}$. Take the crack on the top of the slope as the starting point of the sliding surface, respectively set the toe of the slope as the other end of the sliding surface, the vertical distance of the foot $2 \mathrm{~m}$ as the other end of the sliding surface, and the vertical distance of the slope toe $4 \mathrm{~m}$ as the other end of the sliding surface, Suppose the vertical distance of the slope toe is $8 \mathrm{~m}$ as the other end of the sliding surface, and the vertical distance of the slope toe at $16 \mathrm{~m}$ is the other end of the sliding surface. The sliding surfaces provided are, from bottom to top,sliding surface 1 , sliding surface 2, sliding surface 3, sliding surface 4 , and sliding surface 5, denoted by H1, H2, $\mathrm{H} 3, \mathrm{H} 4$, and H5, respectively. According to formula (13), the stability coefficients of different sliding surfaces can be obtained. The slope related parameters and stability coefficients corresponding to different sliding surfaces are shown in the table 2. Different sliding surface parameters and stability coefficients.

\begin{tabular}{cccc}
\hline NO. & $\boldsymbol{\beta} /\left(^{\circ}\right)$ & $\mathbf{L} / \mathbf{m}$ & $\mathbf{K}$ \\
\hline H1 & 1.94 & 40.04 & 0.88 \\
H2 & 1.90 & 38.46 & 0.89 \\
H3 & 1.84 & 36.92 & 0.90 \\
H4 & 1.70 & 33.98 & 0.93 \\
H5 & 1.29 & 28.89 & 1.11
\end{tabular}

It can be seen from Table 2 that the slope is in an unstable state as a whole and is prone to instability. GEO-STUDIO is widely used in the analysis of slope stability under rainfall conditions, and the operation is simple and the engineering application is strong. In order to verify the results, here we use GEO-STUDIO to simulate the project, as shown in Figure 7.

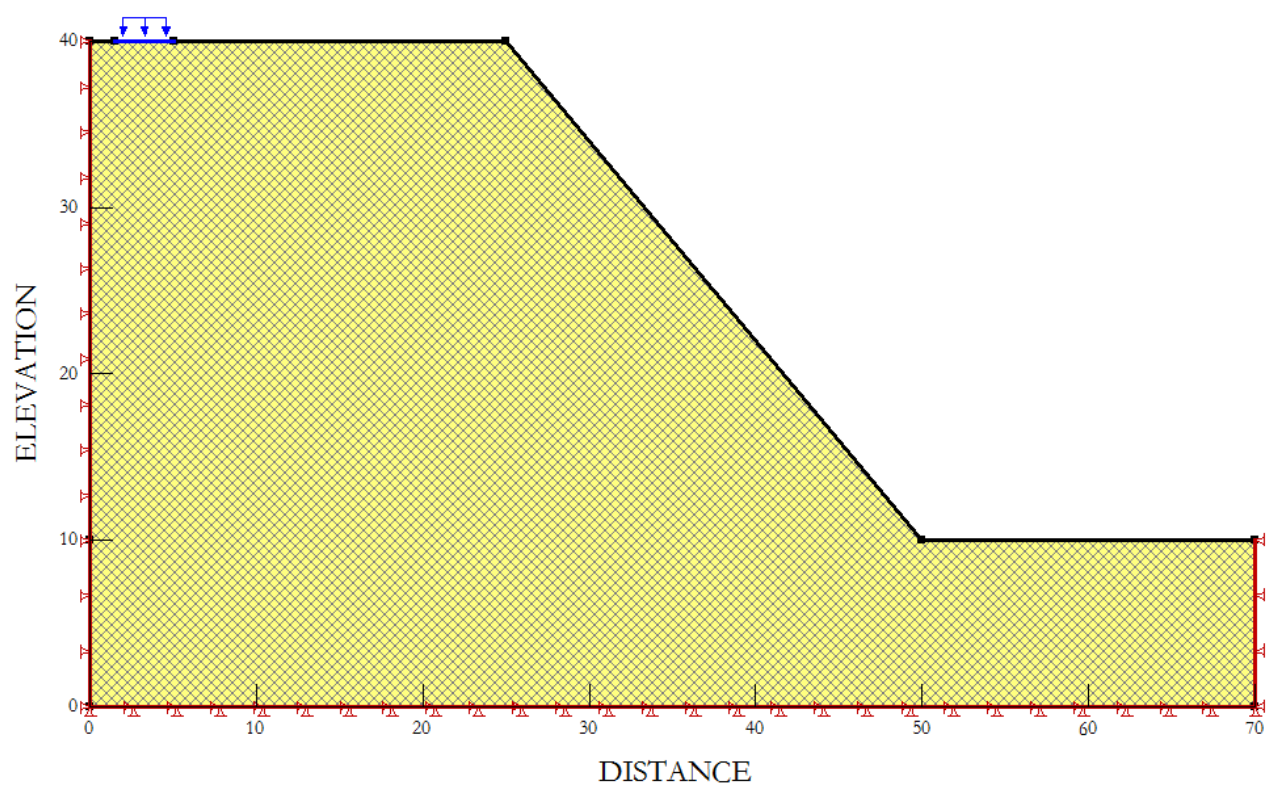

Figure 7. Model built by GEO-STUDIO.

The left and right boundaries of the model are fixed in the $x$ direction, and the bottom boundary of the model is fixed in the xy direction. The calculation results are shown in Table 3. 
Table 3. Comparison of GEO-STUDIO stability coefficient calculation results.

\begin{tabular}{cccccc}
\hline Calculation results & H1 & H2 & H3 & H4 & H5 \\
\hline Improved section method & 0.88 & 0.89 & 0.90 & 0.93 & 1.11 \\
GEOSTUDIO & 0.762 & 0.903 & 0.964 & 1.021 & 1.985 \\
\hline
\end{tabular}

It can be seen from Table 3 that the calculation results of the numerical simulation are basically consistent with the calculation results of the improved strip method. The stability coefficient changes with the sliding surface, showing a trend of increasing from small, and the final judgment result of the slope All are unstable.

\section{Conclusions}

(1) Using elastic theory and Moore Coulomb's theory as transformation conditions, based on the Swedish slice method, the slope stability coefficient solution expression under the slope top load is obtained;

(2) According to the actual engineering, the corresponding slope model structure is established, and 5 sliding surfaces are set with the crack on the top of the slope as the shear outlet. According to the slope stability coefficient expression, the stability coefficient of the set sliding surface is solved, and the stability coefficients are all less than 1.2. The results show that the slope is generally unstable under load, and the judgment result is basically consistent with the GEO-STUDIO calculation result;

(3) In this paper, the sliding surface is assumed to be a straight line. As the thickness of the sliding surface increases, the difference between the calculated results and the actual situation will become larger. Therefore, this method is only used for shallow landslide instability, and the sliding surface will continue to be improved in the future.

\section{References}

1. Chen Chaohui. Stability analysis of building slope under external load. Journal of Hunan City University: Natural Science Edition, 2012, 21,pp. 22-24.

2. Tao Zhigang, Zhao Junkai, Zhang Haijiang, etc. Research on the influence of slope top loading on the stability of gently inclined bedding slopes. Coal Technology, 2017, 36,pp.64-67.

3. Zhihai Zhao,Zhangjian Xu,Yanjie Sun,et al.Influence of Building Load on the Stability of Loess Slope.MMCE2017,WOP in Engineering and Science Research.2017, 5,pp.15-20.

4. Jianlong Yan,Yong Cheng,Yanjie Sun,et al.Influential Factors on the Stability of Building Slope.ESMA2017,Earth and Environmental Science.2017,108.

5. He Siming, Zhang Xiaoxi, Luo Yu. Analysis of potential sliding surface and stability of slope under strip load on top of slope. Journal of Mountain Research, 2011, 29,pp.95-100.

6. Zhang Kegong, Liu Songyu. Soil Mechanics, 4th ed.. Construction Industry Press: Beijing, China , 2016.

7. Li Yunjun, Sun Yanjie, Zhao Zhihai. Improved Felenius method for slope stability evaluation under slope top load. Journal of $X i^{\prime}$ an University of Science and Technology, 2018, 38,: pp.767-775. 